\title{
A7 structure: A family of photonic crystals
}

\author{
C.T. Chan, S. Datta, K.M. Ho, and C.M. Soukoulis \\ Ames Laboratory and Department of Physics and Astronomy, Iowa State University, Ames, Iowa 50011
}

(Received 21 March 1994)

\begin{abstract}
We found that a whole class of structures with rhombohedral symmetry possesses sizable photonic gaps. These structures can be generated by connecting lattice points in an $A 7$ structure by cylinders and a few structures that are known to possess photonic band gaps are in fact members of this "parent" structure. This class of structures also allows us to explore more systematically the criteria favorable for gap formation.
\end{abstract}

The idea that light in ordered dielectric structures could be forbidden from propagating in any direction in certain energy ranges, in analogy with electron waves in semiconductor crystals, was put forward several years ago. ${ }^{1}$ Such a forbidden gap for electromagnetic wave is called a photonic gap, and the periodic dielectric structures that support the photonic gaps are called photonic crystals. The numerous possible applications ${ }^{2}$ of the photonic gap in many different areas have drawn considerable attention from physicists in the past few years. To date, the existence of photonic band gaps in several different two-dimensional ${ }^{3}$ (2D) and 3D (Refs. 4-8) periodic structures have been established both theoretically and experimentally. It is also gratifying that photonic band gaps exist for periodic dielectric structures with refractive index contrasts bigger than $2,4,5$ which is attainable with many materials even in the optical wavelengths. Soon after theory has a handle on this problem, it is quickly realized that very few structures have full photonic gaps for reasonable dielectric contrasts, ${ }^{9}$ and these few seemingly unrelated and isolated 3D structures include the diamond $^{4}$ and diamondlike ${ }^{5}$ structures, the YablonovitchGmitter-Leung (YGL) structure, ${ }^{6}$ the simple cubic structure, ${ }^{7}$ and a face-centered-tetragonal "stacked-bar" structure. ${ }^{8}$ Moreover, there is still one major obstacle that needs to be overcome before the concept of photonic band gap can realize its true potential: The periodic dielectric structure must be fabricable with reasonable ease; otherwise it is unlikely that it can mature into devices or instruments that can have significant impact on science and technology. While it is comparatively easy to fabricate these structures in the microwave length scale, fabricating these $3 D$ structures in the optical length scale poses formidable challenges to current technology. It is thus important to continue with the search for more structures that possess photonic band gaps, since the more examples we have, the better we will understand the systematics of gap formation, and hopefully, some of the new structures may prove more suited for existing or emerging fabrication techniques. In doing so, we found that there is a whole class of structures with rhombohedral symmetry that possesses sizable photonic gaps. Interestingly, most of the structures that are known to date to possess $3 \mathrm{D}$ photonic gaps can be regarded as members of this broader class of $A 7$ structures and are related to each other. Many authors have noted the importance of the connectedness of the structure, and we found that the local connectivity of the structure is also an important factor, and structures composed of the high dielectric component forming a percolating network with the lowest coordination number seem to favor the formation of photonic gaps. We note that some authors used the term connectivity to refer to the connectedness (whether the structure percolates) of the system; here connectivity refers to the number of rods that meet at a particular joint.

A periodic dielectric material ("photonic crystal") can be specified by a position dependent dielectric function $\epsilon(r)=\Sigma_{R, \tau} f(r-R-\tau)$, where $\{R, \tau\}$ defines a periodic lattice. The structure function $f(r)$ can be rather general, but $f(r)$ is usually taken to be a constant $\epsilon_{a}$ at some domain and another constant $\epsilon_{b}$ elsewhere, since these structures are most amenable to fabrication by conventional processes. In this paper, we will consider periodic structures such that the lattice $\{R, \tau\}$ corresponds to that of the $A 7$ crystal structure; ${ }^{10}$ and $f(r)$ corresponds to cylindrical rods with circular cross sections joining the lattice points, preserving the space group symmetry of the $A 7$ crystal structure, and both the high and low dielectrics form percolating networks. Such connected networks are favorable for gap formation. ${ }^{11} \mathrm{We}$ also know from earlier experience ${ }^{5}$ that these percolating structures tend to give the largest gaps with smallest threshold in dielectric contrasts. We considered both dielectric rods in air background, and the inverse structures with air rods in a high dielectric background. In all cases, we have calculated the band gaps for a fixed dielectric constant ratio of 12.96, which corresponds to that of $\mathrm{Si}$ in optical frequencies. We also employ a fixed material filling fraction of $20 \%$, which determines the radius of the rods.

The $A 7$ crystal structure has a rhombohedral lattice and a basis of two atoms. The primitive translation vectors are given by $\vec{a}_{1}=a_{0}\{\epsilon, 1,1\}, \quad \vec{a}_{2}=a_{0}\{1, \epsilon, 1\}, \quad$ and $\vec{a}_{3}=a_{0}\{1,1, \epsilon\} ; \quad$ such that $\epsilon=[1-(1+\cos \alpha$ $\left.\left.-2 \cos ^{2} \alpha\right)^{1 / 2}\right] / \cos \alpha$, and $\alpha$, the shear angle, is the angle between any two $\vec{a}_{i}$ 's. The two basis atoms are positioned at $\vec{d}= \pm u\left(\vec{a}_{1}+\vec{a}_{2}+\vec{a}_{3}\right)$. (For photonic crystals, it is more meaningful to call them "sites" rather than "atoms," and for our structures, these positions are where the rods meet and thus may be called "joints.") The structure can thus be characterized by two parameters: $\alpha$ and $u$. We form the "photonic crystal" by joining the first and second nearest neighbor (NN) sites together by cylinders. By varying $\alpha$ and $u$, we can generate a whole series of "photonic crystals" with very different structural characteristics, but sharing the same symmetry except for some particular values of $u$ and $\alpha$, where the structure attains a higher symmetry. This can happen at $u=0$, 


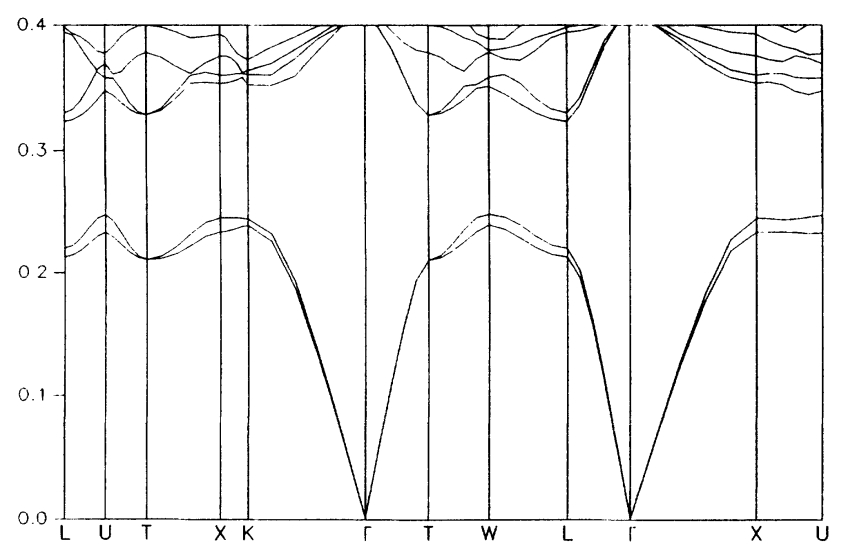

FIG. 1. The photonic band structure for a $A 7$ structure with $\alpha=60^{\circ}$ and $u=1 / 6$, with dielectric rods of $\epsilon=12.96$ in air background. The frequency is given in units of $c / a_{0}$ where $c$ is the speed of light.

$1 / 8$, and $1 / 4$ with $\alpha=60^{\circ}$ or $90^{\circ}$. In these cases, the sudden increase in symmetry will cause a corresponding discontinuous change in the NN lists. We will disregard the higher symmetry for one isolated point in the coordinate space of $\alpha$ and $u$, and just keep the same local connectivity as those structures in the immediate neighborhood of the coordinate space. In these cases, we can imagine that we artificially maintain the lower symmetry of the $A 7$ structure by considering $u \rightarrow u+\delta$, or $\alpha \rightarrow \alpha+\delta$, where $\delta$ is a small number wherever there is a sudden change (increase) in symmetry or connectivity.

We have calculated the photonic bands for a series of structures obtained by varying both $\alpha$ and $u$. We used the plane wave expansion method ${ }^{12}$ with about 1000 plane waves (corresponding to $2000 \times 2000$ matrices for each $\vec{k}$ ). For most of the structures, the gap is determined by calculating $56 k$ points in the Brillouin zone (BZ) boundary. ${ }^{13}$ Photon density of states $^{14}$ are also calculated for a few cases and the gaps found are the same as those determined by just considering points in the BZ boundary. In Fig. 1, we show the photonic band structure for an A7 structure with $\alpha=60^{\circ}$ and $u=1 / 6$. Photonic band gaps are found for a continuous and substantial range of the parameters $\alpha$ and $u$. We show in Fig. 2 the relative size of the photonic gap (measured by the gap to midgap ratio: $\Delta \omega / \omega_{g}$ ) for various values of $u$ at a fixed $\alpha\left(60^{\circ}\right)$, and in Figs. 3(a) and (b), we show the photonic gap for different $\alpha$ for various values of $u$. The structures generated have either fourfold or sixfold connectivity. In some cases, especially for $\boldsymbol{u}>0.16$, there are multiple ways of joining the $A 7$ lattice points by cylinders to create gaps with the same parameters $u$ and $\alpha$, as can be seen in Figs. 2 and 3. For example, at $\alpha \sim 60^{\circ}$, the local connectivity is 4 for $u<0.1875$ and 6 for $u>0.1875$ if we use the stated algorithm to connect the lattice points. For $u>0.1875$, the sixfold connected structure (as defined by our algorithm) has photonic gaps, but we can also obtain photonic gaps if we insist on a fourfold connected structure (Fig. 2). In Figs. 3(a) and (b), we also observe that the sixfold connected $A 7$ structures have gaps at $u \sim 1 / 4$, but if we force a fourfold connectivity (by continuously deforming the $A 7$ structures with smaller $u$ 's at the same $\alpha$, where the natural connectivity is

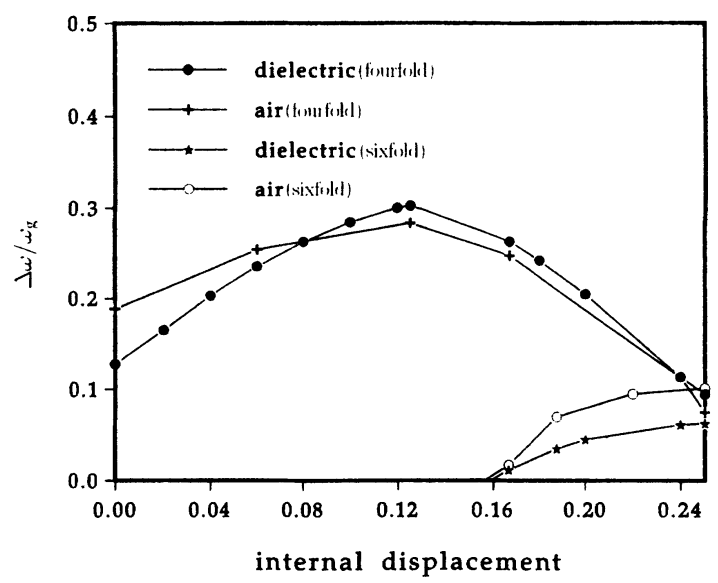

FIG. 2. The relative size of the photonic gap $\left(\Delta \omega / \omega_{g}\right)$ for the $A 7$ structure with different values of the $u$ for $\alpha=60^{\circ}$. Both dielectric rods in air and air cylinders in dielectrics are considered. For $u>0.1875$, the natural connectivity is sixfold, and the fourfold structures are obtained by maintaining the same connectivity as the structures deform continuously from $u<0.1875$ to $u>0.1875$. The lines serve as guides to the eye only.
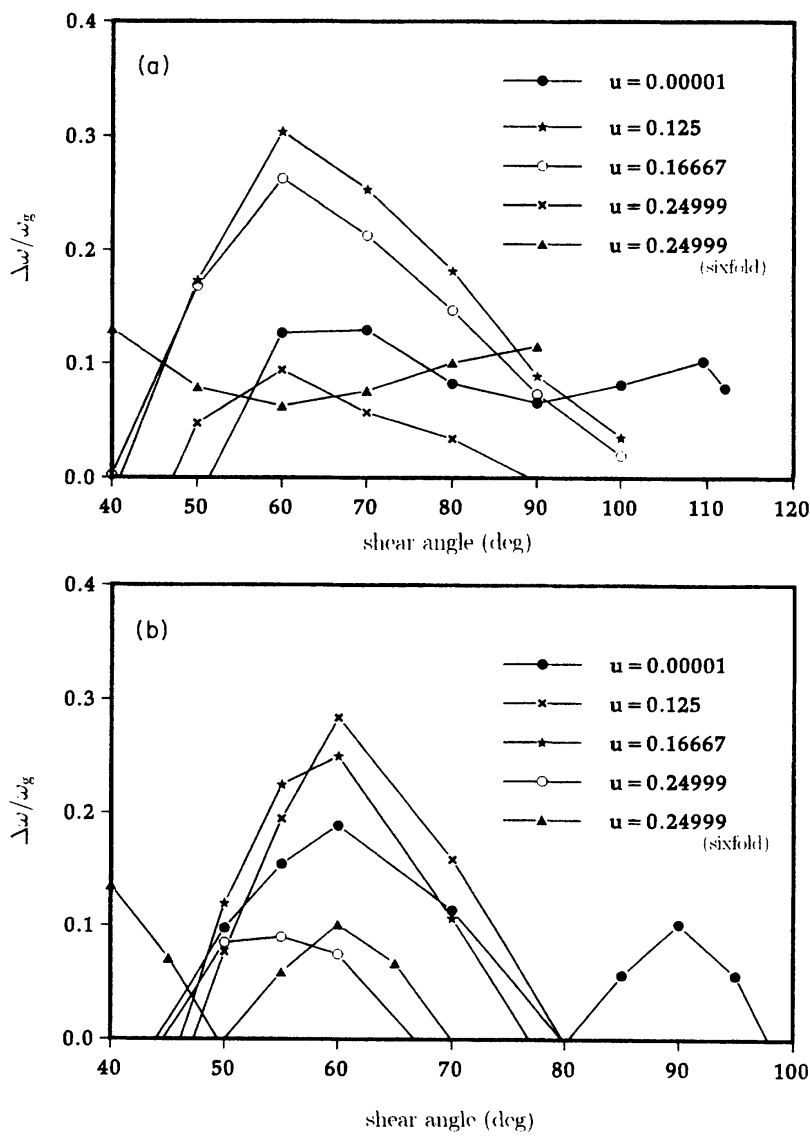

FIG. 3. $\Delta \omega / \omega_{g}$ for the $A 7$ structure with different values of $\alpha=60^{\circ}$ for some values of $u$ : (a) for dielectric rods, and (b) for air rods. The connectivity is fourfold unless indicated by sixfold, where it is sixfold. For $u \sim 1 / 4$, results for both fourfold and sixfold connected structures are shown. 
fourfold), gaps can also be obtained.

It is interesting to note that the few well-known structures that have been reported to have photonic band gaps are in fact members of this class of structures. This includes the diamond structure, ${ }^{4,5}$ which corresponds to $\alpha=60^{\circ}$ and $u$ $=1 / 8$. The region with $u \rightarrow 0$ (i.e., the two "sites" in the $A 7$ fuse together, so that the rod joining them becomes infinitely short) deserves special attention. It exhibits photonic gaps for a continuous range of $\alpha$; and in particular, for $\alpha=60^{\circ}$, it is identical to the YGL structure ${ }^{6}$ (with air rods in high dielectrics), and for $\alpha=90^{\circ}$, it generates a simple cubic structure, ${ }^{7}$ both are known to have photonic gaps. Although not obvious at first glance, the $A 7$ structures with $u \sim 0.25$ are actually structurally equivalent to the $u \sim 0$ structures at a different $\alpha$. In particular, the $A 7$ structure with $\alpha=60^{\circ}$ and $u=0.25$ is also a simple cubic system, but with two sites per unit cell. From Fig. 3(a) (the solid triangles), we see that the simple cubic structure can be deformed to get a bigger gap either by increasing or decreasing $\alpha$.

There are also some members within this class that have very interesting geometry. For example, with $u=1 / 6$, the structure is actually "graphitic," with graphitelike layers formed by rods joining NN sites in a 2D graphite plane, and a fourth rod at each site extending to the nearest neighbor planes. These graphitelike planes are geometrically equivalent to the 2D photonic band gap structures with a triangular lattice of circular holes. ${ }^{3}$ The planes have a stacking sequence of $A B C A B C$. The distance between the layers decreases with increasing $\alpha$. In this sense, the $A 7$ structures with $u=1 / 6$ are "stacked" $2 \mathrm{D}$ photonic band gap structures that have $3 \mathrm{D}$ gaps. The maximum $\Delta \omega / \omega_{g}$ is about $25 \%$ for both air and material rods. The case for $u \rightarrow 0$ and $\alpha$ $=109.47^{\circ}$ is also very interesting. With $\epsilon=-1$, the $\mathrm{BZ}$ has the same shape as that of bcc, and it is equivalent to a bcc structure with 6 out of 8 of the NN joined by rods (except the two neighbors in the [111] direction). A full-symmetry bcc structure does not have photonic gaps in the low lying bands, while this crippled bcc structure with two broken legs has photonic gaps close to $10 \%$ for the case of material rods.

From Figs. 3(a) and (b), which show the variation of the gap with the shear angle $\alpha$ for different $u$, we see that the photonic gaps generally peak at $60^{\circ}$, where the $\mathrm{BZ}$ is most spherical. This reinforces the earlier notion ${ }^{1}$ that isotropic BZ favors gap formations: The spectral gaps in different directions have better chances to overlap if the $\mathrm{BZ}$ is mostly spherical. This is the reason why early experimental works ${ }^{15}$ in a quest for photonic gaps are all focused on the fcc structure. The isotropic $\mathrm{BZ}$ rule, however, is often complicated by the vector nature of light: The gaps for the two possible polarizations in the same direction may work against each other (fcc is a well-known example). ${ }^{12}$ This argument also does not apply when the gap is derived from higher bands, as we can see for the case of sixfold connected material rod structures at $u \sim 1 / 4$ with various $\alpha$ [Fig. 3(a)], and $u \sim 0$ for $\alpha>80^{\circ}$ [Fig. 3(b)].

Another important factor seems to be the local connectivity, or the number of rods joined to a particular site. In general, smaller local connectivity favors large photonic gaps, at least for those cases where the $\mathrm{BZ}$ is reasonably isotropic. If we examine cubic lattices, each site can be fourfold, sixfold, eightfold, or twelvefold coordinated. All the structures here that have gaps are either fourfold or sixfold connected. If we enforce a higher connectivity (but maintain the symmetry) by connecting to more neighbors, the gap disappears. As we have mentioned, a true bcc structure with eight neighbors joined together does not have gaps but the $A 7$ structure with six instead of eight neighbors connected together have photonic gaps. Another example is the fcc structure. If we choose to join up all $12 \mathrm{NN}$ neighbors with rods, there are no gaps in the second and third band (however, a small gap in the higher bands has been reported ${ }^{7}$ ), but the $A 7$ structure here, with the same BZ but a lower connectivity, ${ }^{16}$ a good sized gap is obtained. In Fig. 2, the "natural" connection of the structures is to have a sixfold coordination with $u>0.1875$, but it turns out that fourfold connected structures have bigger gaps. It is interesting to note that for material rods, the simple cubic structure ${ }^{7}$ can achieve a bigger gap by connecting the lattice points in a different manner to reduce the connectivity. In two dimensions, photonic gaps can be obtained by drilling holes in a triangular or a square lattice, and the triangular lattice has larger and more robust gaps than the square lattice. ${ }^{3}$ If we look at the dielectric grid left behind by drilling the holes, the triangular lattice has threefold coordinated sites while the square lattice has fourfold coordinated sites. The triangular lattice also has a more isotropic BZ. This again corroborates the notion that isotropic $\mathrm{BZ}$ and low local connectivity favors photonic gap formation. If we imagine that space is divided into two percolating and disjoint volumes by a connected surface forming complex structures, part of our results can be stated as the structures with the minimum number of genius per joint (such as those bounded by $D$ surfaces and $P$ surfaces ${ }^{17}$ ) have large gaps. In this view, it is not surprising that the diamond structure is still the champion among all known structures since it has the most favorable $\mathrm{BZ}$ and the lowest local connectivity.

The present results also have some immediate implications on fabrication. All $A 7$ air cylinder structures with $u \rightarrow 0$ can be fabricated by drilling three sets of holes into a triangular array, spread out at $120^{\circ}$ on the azimuth, and each hole drilled at an angle $\theta=\cos ^{-1}\left[(\epsilon+2) /\left(\sqrt{3} \sqrt{\epsilon^{2}+2}\right)\right]$ to the normal. The YGL structure, ${ }^{6}$ drilling three sets of holes at $35.3^{\circ}$ to the normal into a triangular lattice, is a special case with $\alpha=60^{\circ}(\epsilon=0)$. From Fig. 3(b) (solid circles), we see that photonic gaps persist for a large range of $\alpha$, and thus photonic gaps can be obtained by drilling all the way from $\theta=30^{\circ}$ to over $55^{\circ}$ (Ref. 18) to the normal. The nature of the gap also changes. It is derived from the second and third band for $\alpha<80^{\circ}$ and from the fifth and sixth band for $\alpha>80^{\circ}$, and thus the midgap frequency $\omega_{g}$ (at which the photonic gap centers) also varies for a fixed filling ratio. This is an extra advantage since the midgap frequency can be tuned simply by varying the drilling angle.

As a summary, we observe that a few photonic crystals known to date, with different structural characteristics and discovered by different authors, are actually related in a broader family of structures. In this family, there are other members with interesting character such as the graphitic structure. Within this "parent" class, we observe that photonic gaps prevail in the lower bands if the structure is a connected network with low local connectivity and has a $\mathrm{BZ}$ that is reasonably isotropic. 
We thank Dr. B.N. Harmon for his comments and suggestions. Ames Laboratory is operated for the U.S. Department of Energy by Iowa State University under Contract No. W-7405-ENG-82. This work was supported by the Director of Energy Research, Office of Basic Energy Sciences, and Advanced Energy Projects including a grant of computer time on the Cray computers at NERSC at the Lawrence Livermore Laboratory.
${ }^{1}$ E. Yablonovitch, Phys. Rev. Lett. 58, 2059 (1987).

${ }^{2}$ See, for example, Photonic Band Gaps and Localization, edited by C.M. Soukoulis (Plenum, New York, 1993); and also, J. Opt. Soc. B 10, 208 (1993).

${ }^{3}$ See, e.g., R.D. Meade et al., Appl. Phys. Lett. 61, 495 (1992); P. Villeneuve and M. Piche, Phys. Rev. B 46, 4969 (1992); M. Philal et al., Opt. Commun. 80, 199 (1991).

${ }^{4}$ K.M. Ho, C.T. Chan, and C.M. Soukoulis, Phys. Rev. Lett. 65, 3152 (1990).

${ }^{5}$ C.T. Chan, K.M. Ho, and C.M. Soukoulis, Europhys. Lett. 16, 563 (1991).

${ }^{6}$ E. Yablonovitch, T.J. Gmitter, and K.M. Leung, Phys. Rev. Lett. 67, 2295 (1991).

${ }^{7}$ H.S. Sozuer, J.W. Haus, and R. Inguva, Phys. Rev. B 45, 13962 (1992); H.S. Sozuer and J.W. Haus, J. Opt. Soc. B 10, 296 (1993).

${ }^{8}$ K.M. Ho et al., Solid State Commun. 89, 413 (1994); E. Ozbay et al., Appl. Phys. Lett. (to be published).

${ }^{9}$ This is in contrast to scalar waves where many structures support full frequency gaps in 3D; see, for example, S. Datta, C.T. Chan, K.M. Ho, and C.M. Soukoulis, Phys. Rev. B 46, 10650 (1992).
${ }^{10} \mathrm{~J}$. Slater, Quantum Theory of Molecules and Solids (McGrawHill, New York, 1965), Vol. 2; L.M. Falicov and S. Golin, Phys. Rev. 137, A871 (1965).

${ }^{11}$ For arguments in 2D, see, for example, R.D. Meade et al., J. Opt. Soc. Am. B 10, 208 (1993).

${ }^{12}$ K.M. Leung and Y.F. Liu, Phys. Rev. Lett. 65, 2646 (1990); Z. Zhang and S. Satpathy, ibid. 65, 2650 (1990); C.T. Chan, K.M. Ho, and C.M. Soukoulis, Mod. Phys. Lett. B 6, 139 (1992).

${ }^{13}$ The reciprocal lattice vectors and the symmetry points in the Brillouin zone of the $A 7$ structure can be found in Ref. 10 .

${ }^{14}$ The density of states are calculated with $231 k$ points in the irreducible $\mathrm{BZ}$ using the linear tetrahedron method.

${ }^{15}$ E. Yablonovitch and T.J. Gmitter, Phys. Rev. Lett. 63, 1950 (1989).

${ }^{16}$ Strictly speaking, it is a fourfold connected structure such that each site has three long and one short bond, but as we take the limit $u \rightarrow 0$, the two lattice points fuse together so that the system is practically a sixfolded connected structure.

${ }^{17}$ A.H. Schoen, Notices Am. Math. Soc. 16, 19 (1969).

${ }^{18} \mathrm{~A}$ simple cubic structure can be formed by drilling at $55^{\circ}$ to the normal has been pointed out to us by Dr. E. Yablonovitch. 